\title{
Role of Immunohistochemistry in Fine Needle Aspiration and Core Needle Biopsy of Thyroid Nodules
}

\author{
Seulki Song ${ }^{1}$ (i) $\cdot$ Hyojin $\mathrm{Kim}^{2} \cdot$ Soon-Hyun Ahn ${ }^{1}$ (i) \\ ${ }^{1}$ Department of Otorhinolaryngology-Head and Neck Surgery, Seoul National University Hospital, Seoul; \\ ${ }^{2}$ Department of Pathology, Seoul National University Bundang Hospital, Seoul National University College of Medicine, Seongnam, Korea
}

Objectives. Immunohistochemistry (IHC) has been used for the diagnosis of indeterminate results in fine needle aspiration (FNA) of thyroid nodules. However, the role of IHC in core needle biopsy (CNB) is not clear and the efficacy of testing for molecular markers following CNB has not been evaluated. The aim of this study is to compare the role of IHC staining in CNB with that in FNA when examining thyroid nodules and to compare the sensitivity and usefulness of different molecular markers.

Methods. Consecutive cases of thyroid FNA and CNB accompanied by IHC from 2004 to 2014 were included in this study with retrospective review of medical record. The rate of remaining nondiagnostic result (unsatisfactory, atypia of undetermined significance or follicular lesion of undetermined significance [AUS/FLUS]) and rate of strong expression of each molecular marker according to the diagnosis were evaluated.

Results. IHC was more frequently performed in CNB with multiple molecular markers compared to FNA (38.1\% vs. $2.8 \%, 3$ or 4 markers [Gal-3, HBME-1, CK19, and CD56] vs. 1 marker [Gal-3]). In the CNB group, 11.3\% remained as AUS/FLUS after IHC, and the rate remaining nondiagnostic was significantly less than in the FNA group (42.9\%). Gal-3 and CK19 showed higher specificity and expressed mainly in conventional type of papillary carcinoma and HBME-1 showed higher sensitivity for the diagnosis of carcinoma with expression in both conventional type and follicular variant papillary thyroid carcinoma.

Conclusion. With these data, we could conclude that IHC was more effective following CNB than following FNA.

Keywords. Thyroid Nodule; Core Needle Biopsy; Fine Needle Aspiration; Immunohistochemistry; Carcinoma

\section{INTRODUCTION}

Thyroid nodules are frequent findings in the general population and are usually diagnosed during routine medical care [1]. Ultrasound-guided fine needle aspiration (FNA) is used as the gold standard initial diagnostic test since it is safe and the most cost-

\footnotetext{
- Received July 1, 2018

Revised October 22, 2018

Accepted October 22, 2018

- Corresponding author: Soon-Hyun Ahn

Department of Otorhinolaryngology-Head and Neck Surgery, Seoul

National University Hospital, 101 Daehak-ro, Jongno-gu, Seoul 03080,

Korea

Tel: +82-2-2072-3649, Fax: +82-2-2072-4057

E-mail: ahnsh30@snu.ac.kr
}

effective diagnostic procedure for thyroid nodules [2,3]. FNA has played a great role in identifying who should be operated on and reducing unnecessary surgery. Nevertheless, $5 \%$ to $17 \%$ of thyroid nodules are not diagnosed correctly and $3 \%$ to $18 \%$ of thyroid nodules are identified as atypia of undetermined significance or follicular lesion of undetermined significance (AUS/ FLUS) [2]. Because of these limitations, repeated FNA or unnecessary surgery may be performed [4]. Although the diagnosis of thyroid nodules is possible through morphological examination with hematoxylin and eosin (H\&E) staining in most cases, evaluation of molecular markers, in cases with thyroid nodules that are difficult to distinguish, has been known to be helpful for differentiating between benign and malignant nodules. Galectin-3 (Gal-3), Hector Battifora mesothelial cell-1 (HBME-1), and

Copyright @ 2019 by Korean Society of Otorhinolaryngology-Head and Neck Surgery

This is an open-access article distributed under the terms of the Creative Commons Attribution Non-Commercial License (http://creativecommons.org/licenses/by-nc/4.0)

which permits unrestricted non-commercial use, distribution, and reproduction in any medium, provided the original work is properly cited. 
cytokeratin-19 (CK19), among other markers, are used for the diagnosis of thyroid nodules $[1,5]$.

Ultrasound-guided core needle biopsy (CNB) has been suggested as an effective complementary tool when indeterminate results are obtained from the initial FNA. However, even with $\mathrm{CNB}$, indeterminate results are still found, and molecular markers can also be examined in CNB specimens. Furthermore, a paraffin block is used after CNB which gives greater opportunities for immunohistochemistry (IHC) staining than after FNA $[2,4]$. The objective of this study is to compare the role of IHC staining in CNB with that in FNA when examining thyroid nodules and to compare the sensitivity and usefulness of different molecular markers.

\section{MATERIALS AND METHODS}

Consecutive cases of FNA or CNB from the ENT outpatient clinic and performed from January 2004 to December 2014 were reviewed retrospectively. The study protocol was approved by the Institutional Review Board of Seoul National University Bundang Hospital (IRB No. B-1505-298-114). Exclusion criteria were non-thyroid disease, for example, parathyroid adenoma and tuberculosis. The procedures of FNA or CNB and cytological or pathological diagnoses were made by experienced radiologists and pathologists. FNA cytopathology was categorized according to The Bethesda System for Reporting Thyroid Cytopathology (TBSRTC) [6]. The results of the CNB were classified according to the suggestions of the Korean Endocrine Pathology Thyroid Core Needle Biopsy Study Group [7]. However, both systems are quite similar and the terminology used here follows TBSRTC guidelines. AUS/FLUS or indeterminate results following FNA and CNB, respectively, are subcategorized into cellular atypia and architectural atypia in this study on the basis of previous work from our department and a meta-analysis which showed a significant difference in the risk of malignancy between cellular and architectural atypia [8,9]. The pattern of ultrasound findings and malignant tumor risks as proposed by the American Thyroid Association guidelines were applied [10]. Of the performed FNA or $\mathrm{CNB}$, only procedures accompanied by additional IHC staining (HBME-1, Gal-3, CK19, CD56, and/or CyclinD1) were included in this study. We performed immunohistochemical staining for the cases of AUS or very small number of atypical cells. The presence of CK19, Gal-3, and HBME-1

\section{H I G H L I G H T S}

- Immunohistochemistry (IHC) has been used for the diagnosis of indeterminate results in thyroid nodules.

- Due to the greater tissue availability of core needle biopsy, the IHC staining procedure was more frequently performed with 3-4 simultaneous markers compared to fine needle aspiration. markers or loss of CD56 markers increases the likelihood of being diagnosed with PTC, so immunohistochemical staining is performed if the tissue is sufficient. The intensity of staining was scored as following: negative (0), focally positive (1, less than $25 \%$ ), and positive ( 2 , more than $25 \%$ ), based on the positive expression of tumor cells. The rate of IHC staining in indeterminate results and conversion of diagnosis was investigated. In this study, nondiagnostic results include unsatisfactory and AUS/ FLUS. Furthermore, nodules that were pathologically confirmed during surgery were collected and analyzed separately.

Data were analyzed using IBM SPSS ver. 20.0 (IBM Corp., Armonk, NY, USA), and the chi-square or Fisher exact test was used for each group comparison. A $P$-value of less than 0.05 was considered significant.

\section{RESULTS}

The study included 490 male and 1,697 female patients with a median age of 50.8 years (range, 9 to 82 years). A total of 2,456 FNA and 679 CNB were performed on 2,406 nodules from 2,187 patients. The rate of nondiagnostic results showed no difference between FNA and CNB group and the total number of procedures that included IHC was 101. IHC was performed in $38.1 \%$ of cases with a nondiagnostic result following CNB, compared with $2.8 \%$ of cases following FNA, a statistically significant difference was observed. The patients in the CNB group were significantly younger and showed suspicious nodules more frequently (Table 1). Fig. 1 shows a flowchart of patients included

Table 1. Demographic data

\begin{tabular}{|c|c|c|c|}
\hline Variable & FNA $(n=21)$ & $\mathrm{CNB}(\mathrm{n}=80)$ & $P$-value \\
\hline Total number & 2,456 & 679 & - \\
\hline Nondiagnostic resulta) & 745 (30.3) & $210(30.9)$ & 0.778 \\
\hline No. of IHC & $21 / 745(2.8)$ & $80 / 210(38.1)$ & $<0.001$ \\
\hline Age (yr) & $57.9 \pm 12.5(34-74)$ & $50.9 \pm 12.7(9-80)$ & 0.027 \\
\hline Male:female & $6: 15$ & $22: 58$ & 0.992 \\
\hline \multicolumn{4}{|l|}{ Nodule size } \\
\hline $\begin{array}{l}\text { Maximum diameter } \\
(\mathrm{mm})\end{array}$ & $6 \pm 3.8(1-14)$ & $4.1 \pm 2.6(1-15)$ & 0.064 \\
\hline $\begin{array}{l}\text { Smallest diameter } \\
(\mathrm{mm})\end{array}$ & $3.5 \pm 1.9(1-7)$ & $2.6 \pm 1.2(1-6)$ & 0.060 \\
\hline ATA risk stratification & & & 0.028 \\
\hline High suspicious & $2(9.5)$ & $24(30.0)$ & \\
\hline $\begin{array}{l}\text { Intermediate } \\
\text { suspicion }\end{array}$ & $7(33.3)$ & $23(28.8)$ & \\
\hline Low suspicion & $10(47.7)$ & $33(41.3)$ & \\
\hline $\begin{array}{l}\text { Very low suspicion, } \\
\text { benign }\end{array}$ & $2(9.5)$ & 0 & \\
\hline
\end{tabular}

Values are presented as number (\%) or mean \pm standard deviation (range). FNA, fine needle aspiration; CNB, core needle biopsy; IHC, immunohistochemistry; ATA, American Thyroid Association.

a)Includes unsatisfactory, atypia of undetermined significance or follicular lesion of undetermined significance. 
in each group. In summary, 11 out of $21(52.4 \%)$ patients in the FNA group and 54 out of $80(67.5 \%)$ patients in the CNB group underwent surgery, with available surgical pathology results.

The reason for IHC staining could be divided into three categories, attempting to overcome an unsatisfactory result, differentiating cellular atypia, and diagnosing architectural atypia. In both groups, IHC was performed more frequently due to architectural atypia. The attempt to overcome an unsatisfactory result following FNA failed in all three cases. After IHC, only nine out of 31 and six out of 67 cases were still classified as cellular atypia and architectural atypia (AUS/FLUS), and the rate of continued nondiagnosis was $29.0 \%$ and $9.0 \%$ respectively. Comparing the efficacy of IHC between FNA and CNB, the rate of continued nondiagnosis was significantly lower in the CNB group ( $11.3 \%$ vs. $42.9 \%)$, meaning that the probability of reclassification was significantly higher in the CNB group (Table 2).

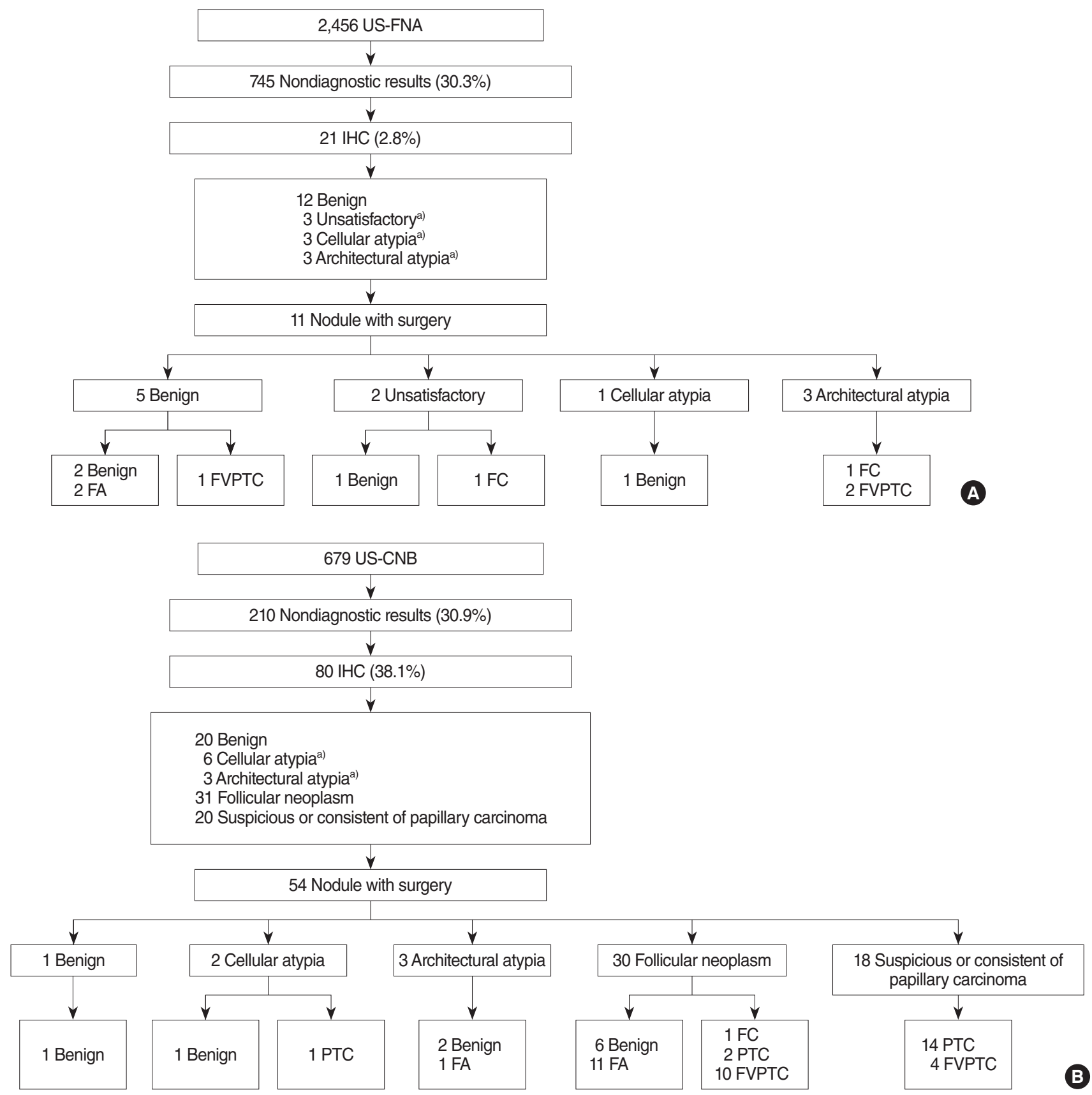

Fig. 1. Flowchart of included cases with immunohistochemical staining $(\amalg \mathrm{HC})$. (A) $I H C$ in fine needle aspiration $(F N A)$ cases. $(B) \amalg H C$ in core needle biopsy (CNB) cases. US-FNA, ultrasound-guided fine needle aspiration; FA, follicular adenoma; FVPTC, follicular variant papillary thy-

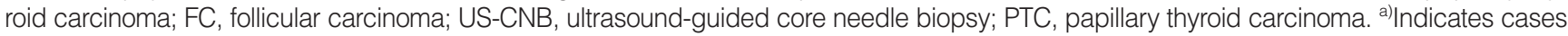
that still had no diagnosis after $\mathrm{HC}$ staining. 
Fig. 2 shows the expression of IHC markers in a heat map. In FNA, due to the paucity of material for IHC, Gal-3 immunostaining alone was performed. However, in the CNB group, Gal3, HBME-1, CK19, and CD56 were simultaneously examined in most cases. There is a tendency that the diagnosis changes to cellular atypia or suspicion of/consistent with PTC if Gal-3 or CK19 is expressed, as shown in Fig. 2A. However, HBME-1 was frequently expressed in follicular neoplasms as well as in papillary carcinoma. Fig. 2B shows the expression of markers according to the surgical pathology; different from the CNB results, HBME-1 is generally expressed in both PTC and FVPTC. In contrast, Gal-3 is expressed mainly in conventional-type PTC. The positive rate of each marker is summarized in Table 3. Gal-3 is specifically expressed in conventional PTC $(70.6 \%)$ and only $41.2 \%$ of FVPTC lesions expressed Gal-3. HBME-1 shows a high rate of expression in all PTC, but about $30 \%$ of benign nodules expressed HBME-1. CK19 is very specific for conventional PTC and CD56 is strongly expressed in benign nodules; however, $30 \%$ of carcinomas also expressed CD56. In summary, Gal-3

Table 2. Purpose of $\mathrm{IHC}$ and the result

\begin{tabular}{|c|c|c|c|}
\hline Reason for IHC & Final diagnosis after IHC & $\begin{array}{c}\text { FNA } \\
(n=21)\end{array}$ & $\begin{array}{c}\text { CNB } \\
(n=80)\end{array}$ \\
\hline \multirow[t]{2}{*}{ Unsatisfactory $(n=3)$} & Unsatisfactory & $3^{\text {a) }}$ & - \\
\hline & Subtotal & 3 & 0 \\
\hline \multirow[t]{4}{*}{ Cellular atypia (n=31) } & Benign & 2 & 6 \\
\hline & Cellular atypia & $3^{\text {a) }}$ & $6^{\text {a) }}$ \\
\hline & $\begin{array}{l}\text { Suspicious or consistent } \\
\text { of PTC }\end{array}$ & 0 & 14 \\
\hline & Subtotal & 5 & 26 \\
\hline \multirow{5}{*}{$\begin{array}{l}\text { Architectural atypia } \\
\quad(n=67)\end{array}$} & Benign & 10 & 14 \\
\hline & Architectural atypia & $3^{\text {a) }}$ & $3^{\text {a) }}$ \\
\hline & FN & 0 & 31 \\
\hline & $\begin{array}{l}\text { Suspicious or consistent } \\
\text { of PTC }\end{array}$ & & 6 \\
\hline & Subtotal & 13 & 54 \\
\hline $\begin{array}{l}\text { Remain nondiagnostic } \\
(\mathrm{no}, \%)^{\mathrm{a})}\end{array}$ & & $9(42.9)$ & $9(11.3)^{b}$ \\
\hline
\end{tabular}

$I H C$, immunohistochemistry; FNA, fine needle aspiration; CNB, core needle biopsy; PTC, papillary thyroid carcinoma; FN, follicular neoplasm. a) Includes unsatisfactory, atypia of undetermined significance or follicular lesion of undetermined significance. ${ }^{\mathrm{b})} \mathrm{P}=0.002$. and CK19 showed high specificity $(96.4 \%$ and $100 \%$, respectively) and HBME- 1 showed high sensitivity of $87.5 \%$ in diagnosing carcinomas. CD56 showed moderate sensitivity and specificity ( $60 \%$ and $72 \%$ ) for diagnosing benign nodules (Table 4 ).

\section{DISCUSSION}

$\mathrm{H} \& \mathrm{E}$ staining is used in the initial histological examination of the thyroid nodule. However, nondiagnostic results after biopsy of thyroid nodule, which cannot be accurately diagnosed using an H\&E stain, present difficulties in determining a patient's management and can result in unnecessary surgery. Therefore, many studies have been carried out on the use of IHC staining applied to thyroid nodules. Studies using HBME- 1 and Gal-3 in combination as IHC markers have shown very high specificity [11-13]. However, using only a single antibody of the IHC panel, accurate diagnosis may be difficult. This is because some studies have shown that Gal-3 is also positive in goiter and thyroiditis, and in a nonnegligible rate of follicular adenomas $[13,14]$. Recently, the advantages of CNB over repeated FNA in a patient with nondiagnostic results in the initial FNA has been reported. This is because CNB can acquire more tissue and can be used to perform microhistologic assessments such as examining follicular structure and the relationship with surrounding tissues better than with FNA $[13,15]$. However, there are few studies of how much nondiagnostic findings can be improved by using IHC staining on CNB specimens. In our study, IHC was significantly more frequently employed in the CNB group com-

Table 4. Sensitivity and specificity of immunohistochemical markers

\begin{tabular}{lrrrr}
\hline Marker & Sensitivity & Specificity & PPV & NPV \\
\hline Diagnosing carcinoma & & & & \\
Gal-3 & 51.4 & 96.4 & 95.0 & 60.0 \\
HBME-1 & 87.5 & 68.2 & 80.0 & 78.9 \\
CK19 & 51.5 & 100.0 & 100.0 & 57.9 \\
Diagnosing benign & & & & \\
CD56 & 60.0 & 72.0 & 63.2 & 69.2 \\
\hline
\end{tabular}

PPV, positive predictive value; NPV, negative predictive value; Gal-3, galectin-3; HBME-1, Hector Battifora mesothelial cell-1; CK19, cytokeratin-19.

Table 3. Positive expression (>25\%) rate of immunohistochemical markers according to the surgical pathology

\begin{tabular}{lcccr}
\hline Variable & Galectin 3 & HBME1 & CK19 & CD56 \\
\hline Benign $(n=14)$ & $1 / 14(7.1)$ & $3 / 10(30.0)$ & $0 / 10$ & $7 / 8(87.5)$ \\
FA $(n=14)$ & $0 / 14$ & $4 / 12(33.3)$ & $0 / 12$ & $0 / 12(41.7)$ \\
FC $(n=3)$ & $0 / 3$ & $1 / 1(100)$ & $0 / 2$ & $0 / 1$ \\
FVPTC $(n=17)$ & $7 / 17(41.2)$ & $12 / 14(85.7)$ & $5 / 14(35.7)$ & $4 / 14(28.6)$ \\
PTC $(n=17)$ & $12 / 17(70.6)$ & $15 / 17(88.2)$ & $12 / 17(70.6)$ & $3 / 10(30.0)$ \\
Total $(n=65)$ & $20 / 65(30.8)$ & $35 / 54(77.8)$ & $17 / 55(30.9)$ & $19 / 45(42.2)$ \\
\hline
\end{tabular}

Values are presented as number (\%).

FA, follicular adenoma; FC, follicular carcinoma; FVPTC, follicular variant papillary thyroid carcinoma; PTC, papillary thyroid carcinoma. 

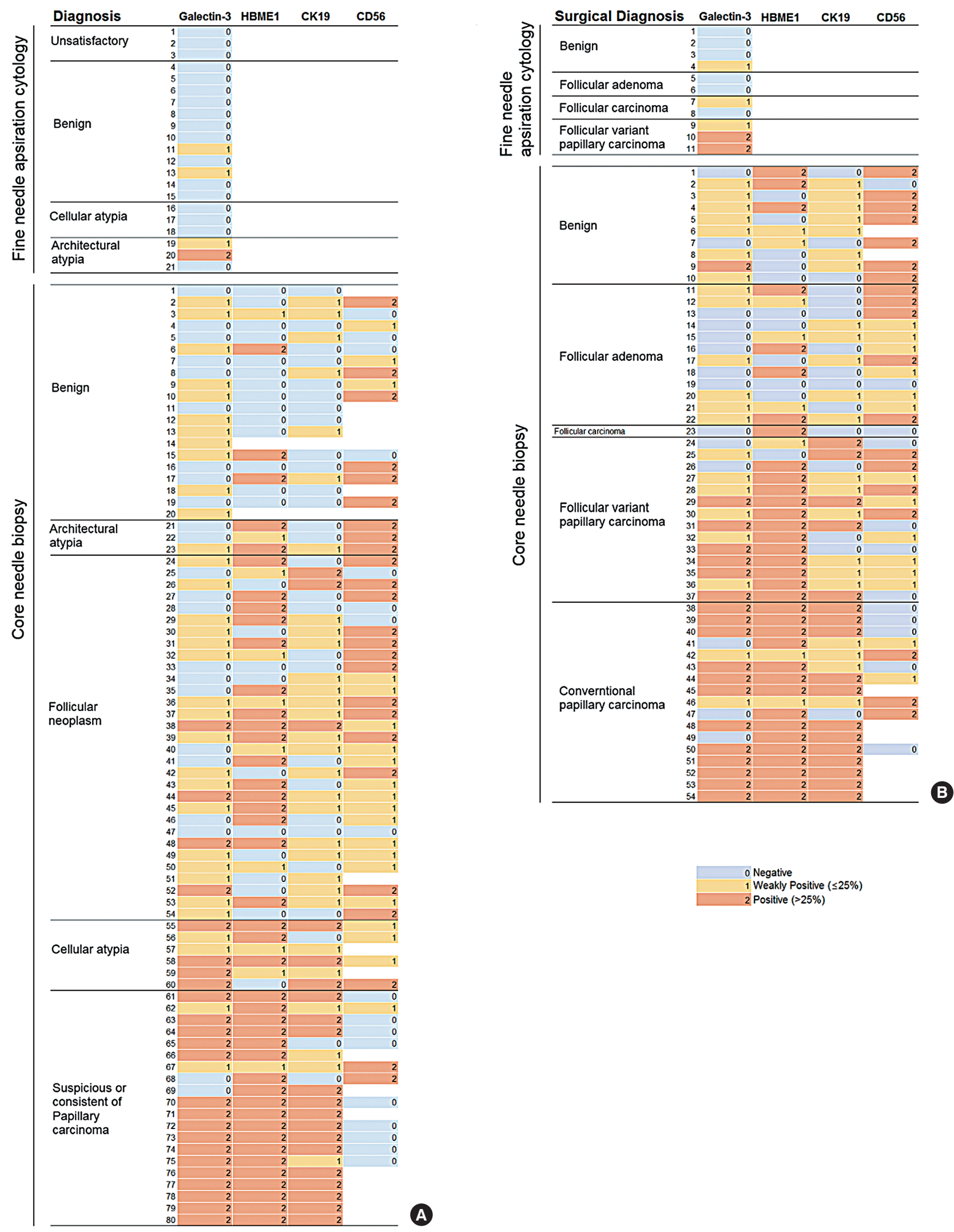

Fig. 2. Heat map of expression of each molecular marker following fine-needle aspiration or core needle biopsy. (A) Expression according to preoperative diagnosis after immunohistochemistry. (B) Expression according to the surgical pathology. 
pared to the FNA group. Furthermore, although only Gal-3 was examined in FNA group, three or more markers could be examined simultaneously in the CNB group. This suggests that the amount of tissue from FNA is insufficient to carry out IHC staining.

Considering the efficacy of IHC with respect to its purpose, attempting to resolve issues with unsatisfactory specimens was unsuccessful in the FNA group. As unsatisfactory findings are quite rare when using $\mathrm{CNB}$, there were no such cases in the $\mathrm{CNB}$ group. In differentiating cellular atypia, $71 \%(22 / 31)$ could be reclassified as a diagnostic result. In terms of architectural atypia, 9\% (6/67) remained classified as architectural atypia; thus, IHC appears quite effective in differentiating diagnoses. Differentiating between follicular adenoma and carcinoma was not attempted in our institute, and 31 patients were diagnosed with follicular neoplasm after IHC. There may be debate whether a diagnosis of follicular neoplasm is diagnostic and whether these patients benefitted from the use of IHC in their diagnosis. Overall, the rate of findings that remained unsatisfactory or AUS/FLUS after IHC was significantly lower in the CNB group and we concluded that IHC is more informative in CNB. In the CNB group, $67.5 \%$ (54/80) of IHC was performed in cases with an initial finding of architectural atypia. In our previous study, the consecutive analysis of biopsy results from thyroid nodules showed a significantly lower rate of cellular atypia and significantly higher diagnostic rate of architectural atypia or follicular neoplasm after performing CNB in nodules with intermediate or low suspicious features [16]. This may be the reason for the frequent use of IHC when architectural atypia is found using CNB.

Gal-3 and CK19 showed high specificity and HBME-1 showed high sensitivity for carcinomas. In another study, Gal-3 expression is reported to have $100 \%$ sensitivity and $94 \%$ specificity for distinguishing follicular adenoma from follicular carcinoma [17]. However, in our study, the expression of Gal-3 in follicular carcinoma or FVPTC was not as frequent $(0 \%$ and $41.2 \%)$. Therefore, Gal-3 is useful for the diagnosis of conventional-type papillary thyroid carcinoma rather than FVPTC based on our data. CK19 showed $100 \%$ specificity but only a $35.7 \%$ positive findings rate in FVPTC. Gal-3 and CK19 also exhibited a high coincidence rate. In contrast, HBME-1 was expressed in over $85 \%$ of conventional PTC and FVPTC. As HBME- 1 is also expressed in $30 \%$ of benign lesions, HBME-1 will have more use in screening rather than diagnosis.

A limitation of this retrospective study is that the selection of cases was determined by an individual pathologist and not by uniform indication. Therefore, the potential for selection bias is present. Furthermore, not all four markers were used in all procedures and IHC staining following FNA was limited to only Gal-3, limiting the effectiveness of comparing each marker.

Due to the greater tissue availability of CNB, the IHC staining procedure was more frequently performed with 3-4 simultaneous markers compared to FNA. The rate of continued non- diagnostic results was significantly lower in the CNB group than in the FNA group. As Gal-3 and CK19 showed high specificity and HBME-1 showed high sensitivity; these markers have complementary uses in the diagnosis of nondiagnostic nodules.

\section{CONFLICT OF INTEREST}

No potential conflict of interest relevant to this article was reported.

\section{ORCID}

Seulki Song https://orcid.org/0000-0001-5791-2607

Soon-Hyun Ahn https://orcid.org/0000-0002-0759-6850

\section{REFERENCES}

1. Nechifor-Boila A, Catana R, Loghin A, Radu TG, Borda A. Diagnostic value of HBME-1, CD56, Galectin-3 and Cytokeratin-19 in papillary thyroid carcinomas and thyroid tumors of uncertain malignant potential. Rom J Morphol Embryol. 2014;55(1):49-56.

2. Na DG, Kim JH, Sung JY, Baek JH, Jung KC, Lee H, et al. Coreneedle biopsy is more useful than repeat fine-needle aspiration in thyroid nodules read as nondiagnostic or atypia of undetermined significance by the Bethesda system for reporting thyroid cytopathology.Thyroid. 2012 May;22(5):468-75.

3. Doddi S, Chohda E, Maghsoudi S, Sheehan L, Sinha A, Chandak P, et al.The final outcome of indeterminate cytology of thyroid nodules in a District General Hospital. G Chir. 2015 May-Jun;36(3):122-7.

4. Na DG, Baek JH, Jung SL, Kim JH, Sung JY, Kim KS, et al. Core needle biopsy of the thyroid: 2016 consensus statement and recommendations from Korean Society of Thyroid Radiology. Korean J Radiol. 2017 Jan-Feb;18(1):217-37.

5. Saleh HA, Jin B, Barnwell J, Alzohaili O. Utility of immunohistochemical markers in differentiating benign from malignant follicularderived thyroid nodules. Diagn Pathol. 2010 Jan;5:9.

6. Choi YJ, Baek JH, Suh CH, Shim WH, Jeong B, Kim JK, et al. Coreneedle biopsy versus repeat fine-needle aspiration for thyroid nodules initially read as atypia/follicular lesion of undetermined significance. Head Neck. 2017 Feb;39(2):361-9.

7. Jung CK, Min HS, Park HJ, Song DE, Kim JH, Park SY, et al. Pathology reporting of thyroid core needle biopsy: a proposal of the Korean Endocrine Pathology Thyroid Core Needle Biopsy Study Group. J Pathol Transl Med. 2015 Jul;49(4):288-99.

8. Ahn SH, Kim SD, Jeong WJ. Comparison of risk of malignancy in a subgroup with atypia of undetermined significance/follicular lesion of undetermined significance: a meta-analysis. Head Neck. 2017 Aug;39(8):1699-710.

9. Kim SD, Han SH, Jeong WJ, Kim H, Ahn SH. Differences in clinical features between subcategories of "atypia/follicular lesion of undetermined significance". Endocr Pathol. 2017 Sep;28(3):247-52.

10. Li XY, Zhang B, Lin YS. The interpretation of 2015 American Thyroid Association management guidelines for adult patients with thyroid nodules and differentiated thyroid cancer. Zhonghua Er Bi Yan Hou Tou Jing Wai Ke Za Zhi. 2017 Apr;52(4):309-15.

11. Saggiorato E, De Pompa R, Volante M, Cappia S, Arecco F, Dei Tos 
AP, et al. Characterization of thyroid 'follicular neoplasms' in fineneedle aspiration cytological specimens using a panel of immunohistochemical markers: a proposal for clinical application. Endocr Relat Cancer. 2005 Jun;12(2):305-17.

12. Fadda G, Rossi ED, Raffaelli M, Pontecorvi A, Sioletic S, Morassi F, et al. Follicular thyroid neoplasms can be classified as low- and highrisk according to HBME-1 and Galectin-3 expression on liquid-based fine-needle cytology. Eur J Endocrinol. 2011 Sep;165(3):447-53.

13. Trimboli P, Guidobaldi L, Amendola S, Nasrollah N, Romanelli F, Attanasio D, et al. Galectin-3 and HBME-1 improve the accuracy of core biopsy in indeterminate thyroid nodules. Endocrine. 2016 Apr; 52(1):39-45

14. Chiu CG, Strugnell SS, Griffith OL, Jones SJ, Gown AM, Walker B, et al. Diagnostic utility of galectin-3 in thyroid cancer. Am J Pathol.
2010 May;176(5):2067-81.

15. Trimboli P, Nasrollah N, Guidobaldi L, Taccogna S, Cicciarella Modica DD, Amendola S, et al. The use of core needle biopsy as first-line in diagnosis of thyroid nodules reduces false negative and inconclusive data reported by fine-needle aspiration. World J Surg Oncol. 2014 Mar;12:61.

16. Ahn SH, Park SY, Choi SI. Comparison of consecutive results from fine needle aspiration and core needle biopsy in thyroid nodules. Endocr Pathol. 2017 Dec;28(4):332-8.

17. Bartolazzi A, Gasbarri A, Papotti M, Bussolati G, LucanteT, Khan A, et al. Application of an immunodiagnostic method for improving preoperative diagnosis of nodular thyroid lesions. Lancet. 2001 May;357(9269):1644-50. 